bioRxiv preprint doi: https://doi.org/10.1101/639278; this version posted June 25, 2019. The copyright holder for this preprint (which was not certified by peer review) is the author/funder, who has granted bioRxiv a license to display the preprint in perpetuity. It is made available under aCC-BY-NC-ND 4.0 International license.

\title{
Actin chromobody imaging reveals sub-organellar actin dynamics
}

Cara R. Schiavon ${ }^{1 *}$, Tong Zhang ${ }^{1 *}$, Bing Zhao ${ }^{2}$, Leonardo Andrade ${ }^{1}$, Melissa Wu ${ }^{1}$, Tsung-Chang Sung ${ }^{3}$, Yelena Dayn ${ }^{3}$, Jasmine W. Feng ${ }^{4}$, Omar A. Quintero ${ }^{4}$, Robert Grosse ${ }^{3}$, Uri Manor ${ }^{1 \S}$

1. Waitt Advanced Biophotonics Center, Salk Institute for Biological Studies, USA

2. Institute of Experimental and Clinical Pharmacology and Toxicology, University of Freiburg, Germany

3. Transgenic Core, Salk Institute for Biological Studies, USA

4. Department of Biology, University of Richmond, USA

*Contributed equally

§Corresponding author: umanor@salk.edu

\section{Abstract}

The actin cytoskeleton plays multiple critical roles in cells, from cell migration to organelle dynamics. The small and transient actin structures regulating organelle dynamics are difficult to detect with fluorescence microscopy. We developed an approach using fluorescent protein-tagged actin nanobodies targeted to organelle membranes to enable live cell imaging of previously undetected sub-organellar actin dynamics with high spatiotemporal resolution. These probes reveal that ER-associated actin drives fission of multiple organelles including mitochondria, endosomes, lysosomes, peroxisomes, and the Golgi.
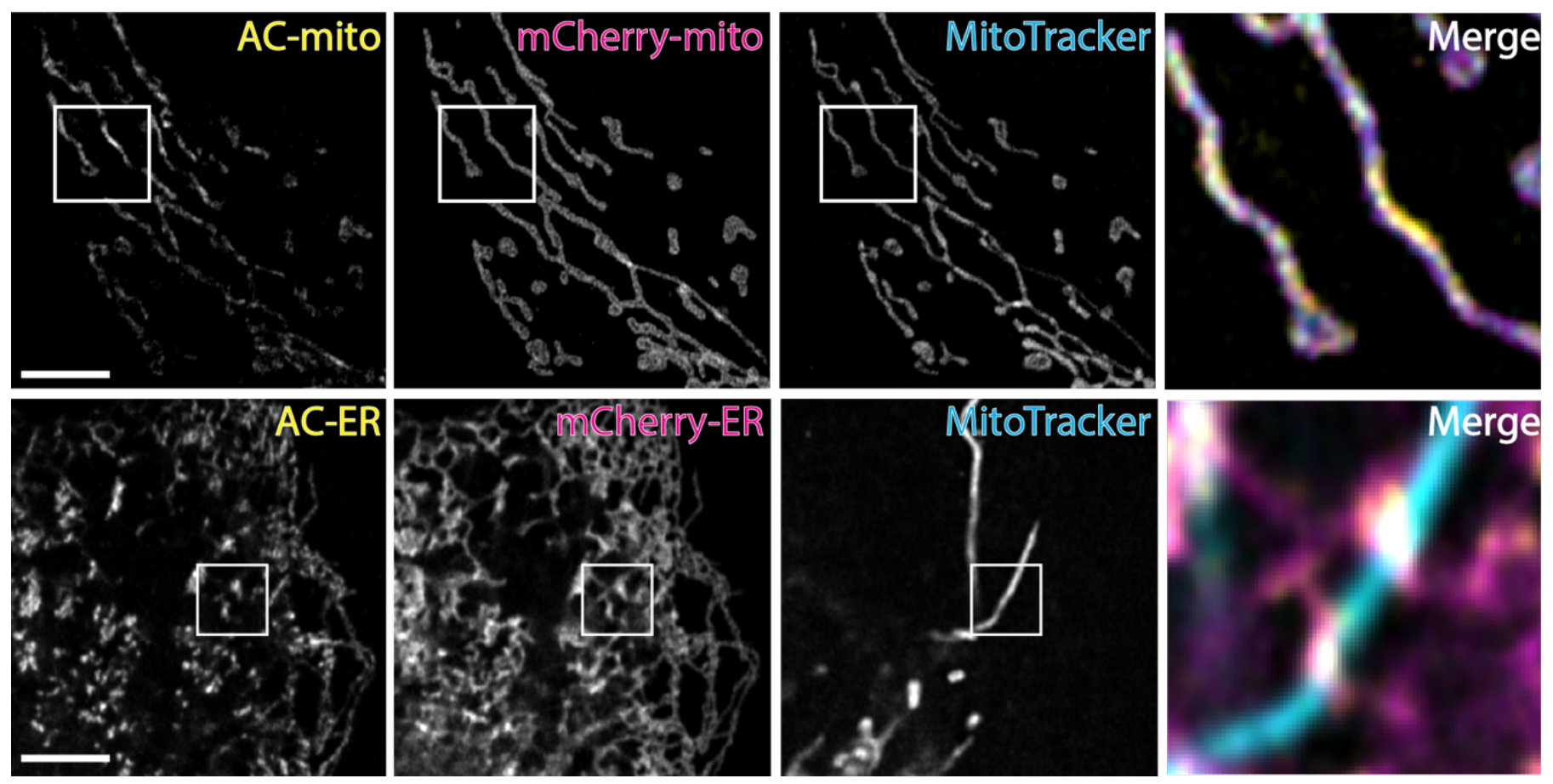


\section{Introduction}

The critical role of the actin cytoskeleton in organelle dynamics is largely accepted, but poorly understood. The precise spatiotemporal dynamics of actin at organelle membranes remain particularly unclear due to the combined limitations of currently available actin probes and imaging approaches. For fluorescence microscopy approaches, imaging smaller actin structures in the cell suffers from an enormous background signal issue - the high signal from the dense meshwork of actin filaments throughout the cell overwhelms the signal from the relatively small, transient actin structures associated with organelle dynamics. Furthermore, the limitations in resolution make it difficult to determine whether any actin filaments are physically associated with the organelle. Meanwhile, EM approaches can be used to visualize actin filaments with extremely high resolution ${ }^{1,2}$. However, these techniques only capture single timepoints, making it difficult to determine the precise state or dynamics of the actin filaments or their associated organelles at the time of fixation. All these limitations ultimately preclude a solid understanding of the mechanisms by which actin regulates organelle dynamics in health or disease. Here we employ fluorescent protein-tagged actin nanobodies, aka "actin chromobodies" $(\mathrm{AC})^{3,4}$, fused to organelle membrane targeting sequences to facilitate live cell imaging of sub-organellar actin dynamics with high spatiotemporal resolution. Using these probes we discovered that ER-associated actin accumulates at all ER-organelle contacts during organelle fission.

\section{$\underline{\text { Results }}$}

We hypothesized that AC probes with organelle membrane targeting sequences could be used to monitor actin dynamics exclusively within a $\sim 10 \mathrm{~nm}$ distance from the target organelle membrane. Given the high mobility of membrane anchored proteins in a bilayer, an F-actin binding probe containing only a membrane targeting sequence and a fluorescent protein tag should quickly accumulate at sites of F-actin enrichment near the membrane (Figure 1a). To test this hypothesis, we generated constructs containing the yeast Fis1 mitochondrial outer membrane and Cytb5ER endoplasmic reticulum (ER) minimal C-terminus tail membrane targeting sequences fused to the cytoplasm-facing actin

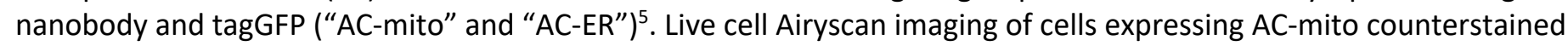
with MitoTracker dye revealed strikingly specific regions of AC-mito enrichment on the surfaces of mitochondria (Figure $\underline{1 b}$ and Supplementary Movies 1-2). Similarly, AC-ER expressing cells revealed significant accumulation of AC-ER at specific ER-mitochondria contact sites (Figure 1b and Supplementary Movies 3-4). To rule out the possibility that the membrane targeting sequences we used were causing the probe to accumulate in specific regions independent of actinbinding activity, we co-transfected AC-mito or AC-ER with mCherry-tagged versions of the mitochondrial (Fis1) and ER (Cytb5ER) membrane targeting sequences (mCherry-mito and mCherry-ER). As expected, the mCherry-mito and mCherry-ER signals were evenly distributed along their respective organelle membranes, with no obvious enrichment in any specific regions. In contrast, the co-expressed AC-mito and AC-ER constructs were uniquely enriched in specific regions on their respective organelles (Figure 1b). mCherry-ER showed some accumulation at ER-mitochondria intersections, indicative of stable contacts at these sites ${ }^{6}$. However, the AC-ER probe showed particularly enhanced enrichment at ER-mitochondria contacts, revealing significant accumulation of actin specifically at these contact sites (Figure 1b). Fluorescence recovery after photobleaching (FRAP) experiments showed that both the AC and mCherry probes are highly mobile on the membrane, but the AC probes exhibit significantly lower mobility and a higher immobile fraction (Figure 1c, Supplementary Movies 5-6), consistent with the model in which a fraction of AC probes are immobilized by actin (Figure 1a).

In our hands, pan-actin probes such as phalloidin fail to clearly reveal sites of actin accumulation on mitochondria or the ER under normal imaging conditions (Supplementary Figure 1). Furthermore, given the abundance of actin signal throughout the cell and the limitations of resolution in fluorescence microscopy, it is extremely difficult to assess whether colocalization of a pan-actin signal and an organelle membrane is a true actin-organelle association or not. Since our AC probes are small proteins tethered to their respective organelle membranes, we can conclude that all observed actin accumulation is within a maximum distance of $10 \mathrm{~nm}$ from the organelle membrane (although the distal ends of associated actin filaments may extend far beyond 10nm). Using our AC probes as a guide, we were able to identify regions of actin accumulation using a cytoplasmic pan-actin probe (i.e. AC-tagRFP) (Figure 1d). Since phalloidin is widely considered the gold standard for F-actin labeling ${ }^{4}$, we attempted to colocalize AC-mito and AC-ER with phalloidin staining in fixed cells (Supplementary Figure 2). Interestingly, we found that fixation with 4\% PFA causes a dramatic loss in AC-mito enrichment as well as an overall decrease in AC-mito or AC-ER signal, perhaps reflective of the relatively small and transient nature of these membrane-associated actin structures. However, we were still able to detect coaccumulation of our probes with phalloidin in fixed cells. Notably, the contrast in the phalloidin signal needed to be 
extremely high in order to visualize the signal at AC enriched sites (Supplementary Figure 2), causing neighboring structures to be completely saturated or clipped. We next tested whether other fixation methods could both improve preservation of the AC probe signal as well as phalloidin labeling of AC accumulation sites. Inspired by recent work in the Svitkina lab $^{1}$, we found that using a cytoskeletal stabilization buffer and light saponin-mediated membrane extraction better preserved the AC signal and improved phalloidin labeling at AC-mito/ER enrichment sites (Supplementary Figure 2). These results highlight the value of live imaging labeling approaches and show why similar actin enriched sites were not previously detected with standard phalloidin staining and fluorescence imaging. Indeed, only with the AC-mito/ER signal serving as a guide could we confidently identify sub-organellar regions of actin enrichment in our phalloidin signal.

We considered it important to determine whether the Fis1 membrane targeting sequence in our AC-mito probe might induce dominant negative effects on mitochondrial dynamics, perhaps by outcompeting endogenous Fis1 for mitochondrial outer membrane localization. Since the AC-mito construct uses only the C-terminal membrane targeting domain of Fis1, an anti-Fis1 antibody against the cytoplasmic N-terminal domain should only detect endogenous protein. We found no change in endogenous Fis1 localization to mitochondria in AC-mito expressing cells compared to neighboring non-transfected cells (Supplementary Figure 3). Finally, to determine whether our localization results were specific to the Fis1 membrane targeting sequence or to actin nanobodies in particular, we generated variants of our ACmito probe using a different membrane targeting sequence (Cytb5mito ${ }^{5}$ ) and/or a different F-actin probe (LifeAct ${ }^{7}$ ). All four of our probes yielded very similar results, revealing specific sub-mitochondrial regions of actin accumulation (Supplementary Figure 4). However, the LifeAct probes displayed a more diffuse pattern on the mitochondrial outer membrane. In contrast, our AC probe seemed to better highlight specific subdomains of actin enrichment, consistent with previous comparative studies suggesting that actin nanobodies have superior F-actin labeling capabilities ${ }^{4}$. Thus, we decided to mainly use our AC probes for the majority of our experiments. To further test whether AC-mito accumulation on mitochondria is dependent on F-actin, we treated AC-mito expressing cells with the F-actin depolymerizing drug Latrunculin $B$ (LatB), which significantly reduced the AC-mito signal. Most of the remaining signal appeared as punctate structures, matching the punctate F-actin structures observed with the pan-actin probe AC-tagRFP ("pan-AC") (Supplementary Figure 5). Importantly, LatB treatment resulted in a more diffuse distribution of AC-mito, reflective of a reduction of F-actin available on or near mitochondria to induce accumulation. Similar results were observed after treatment with cytochalasin D (Supplementary Movie 7). Taken together, these results strongly support the conclusion that our AC probes are labeling F-actin on their respective membrane surfaces. These probes thus facilitate unprecedented resolution (10nm from the membrane) and signal-to-background imaging of mitochondria- and ERassociated actin filaments in live cells. It should be noted that most (but not all - see Moore et al. ${ }^{8}$ ) previous live imaging of actin and mitochondria was performed during overexpression or stress conditions in order to increase mitochondrial fission and/or the associated actin signal at mitochondria ${ }^{9-14}$, whereas these data were obtained under normal physiological conditions.

Previous studies have demonstrated a role for ER-mitochondria contact sites in driving mitochondrial fission, likely with actin playing a role in force generation and Drp1 recruitment and activation ${ }^{1,9-15}$. However, due to the abundance of actin in the cytoplasm, it is difficult to detect actin accumulating near ER-mitochondria contact sites, or whether any such actin directly associates with either organelle. Furthermore, pharmacological or genetic perturbation of actin dynamics likely causes global changes to the entire cell, making it difficult to distinguish direct versus indirect effects. To determine whether mitochondria- or ER-associated actin accumulates during ER-mediated fission, we performed live imaging of cells co-expressing AC-mito and mCherry-tagged Drp1, an actin-binding dynamin-related GTPase protein essential for mitochondrial fission ${ }^{10,16,17}$ (Figure 2, Supplementary Figure 6, and Supplementary Movies 8-11). To avoid potential overexpression artifacts, we used the low expression ubiquitin promoter $C$ to drive expression of our $A C$ probes ${ }^{18}$. We found AC-mito accumulates at or immediately adjacent to mitochondrial fission sites prior to Drp1mediated fission (Figure 2a, Supplementary Figure 6a, Supplementary Movie 8). Interestingly, we frequently observed elongated regions of AC-mito enrichment crossing over mitochondrial constriction sites, consistent with recent reports of similar actin structures observed with platinum replica electron microscopy of membrane extracted cells ${ }^{1}$. We similarly observed accumulation of AC-ER at mitochondrial fission sites prior to Drp1-mediated fission (igire 2b, Supplementary Figure 6b, Supplementary Movie 9). Co-expression of AC-mito or AC-ER with mCherry-ER showed that both mitochondria- and ER-associated actin accumulates at ER-tubules marking mitochondrial fission sites (Figure 2c-d, Supplementary Figure 6c-d, Supplementary Movies 10-11). Finally, co-expression of AC-mito and AC-ER revealed a 
consistent pattern wherein mitochondrial-associated actin accumulates first, followed by ER-associated actin prior to fission (Figure 2e, Supplementary Figure 6e, Supplementary Movie 12). These results clarify the spatiotemporal dynamics of a subpopulation of actin filaments specifically associated with the mitochondrial outer membrane versus the ER prior to and during mitochondrial fission.

Recent studies have suggested an analogous role for the ER in mediating endosomal fission ${ }^{19,20}$. However, direct evidence for ER-associated actin during fission is missing. Using our AC-ER probe, we observed actin enrichment at ERassociated actin at endosomal constriction and fission sites (Figure 3b, Supplementary Movie 14). Given the ER regularly contacts most organelles in the cell more than $90 \%$ of the time ${ }^{21}$, we hypothesized that actin accumulates at all ERorganelle contact sites to drive their constriction as a preparatory step for fission. Live imaging of cells co-expressing ACER and markers for mitochondria, endosomes, peroxisomes, lysosomes, and the Golgi all revealed accumulation of ERassociated actin at fission sites for all of these organelles (Figure 3a-e, Supplementary Movies 13-17). Importantly, the frequency of AC-ER probe accumulation was significantly greater than what would be expected by chance

(Supplementary Figure 7). Scanning electron microscopy (SEM) imaging of cells treated with saponin and cytoskeleton stabilization buffer shows actin filaments associated with mitochondria in similar patterns observed with AC-mito (Figure 3f). Finally, AC-ER reveals a striking network of ER-associated actin bundles on the nucleus (Figure 3g, Supplementary Movie 18). SEM imaging of non-transfected cells treated with saponin and cytoskeleton stabilization buffer shows a similar network of actin fibers accumulating on the nucleus, validating the localization pattern observed in our AC-ER fluorescence data (Figure 3g). A perinuclear actin cap structure has been previously reported and is known to have important roles in multiple cellular processes ${ }^{22-27}$. However, as with other actin structures, the actin filaments specifically associated with the nucleus have been difficult to visualize with pan-actin probes due to the overwhelming signal from surrounding actin stress fibers and cortical actin. Thus, AC-ER labeling provides a more specific labelling approach for visualization of perinuclear actin structures.

\section{Conclusion}

Taken together, our data reveal a novel role for the ER in recruiting actin filaments to all ER-organelle membrane contact sites, most likely in order to drive membrane remodeling and scaffolding for dynamin-mediated membrane scission. Given the important role of both organelle-organelle contact sites and the actin cytoskeleton in health and disease, the apparent ability of the ER to drive actin accumulation at organelle contacts has broad implications for both cell biology and biomedical research. Finally, the ability to use membrane-anchored AC probes as "proximity sensors" for subcellular actin dynamics provides a novel tool for studying the role of actin in a wide range of cell biological processes. The variety of subcellular compartments, protein targets, and corresponding subcellular or sub-organellar processes that can be studied will increase as additional nanobodies and targeting sequences are developed.

\section{Methods}

Cell culture. U2OS and Hap1 cells were purchased from ATCC. Cells were grown in DMEM supplemented with $10 \%$ fetal bovine serum at $37^{\circ} \mathrm{C}$ with $5 \% \mathrm{CO}_{2}$. Cells were transfected with Lipofectamine 2000 (ThermoFisher). Cells were plated onto either 8-well \#1.5 imaging chambers or \#1.5 35mm dishes (Cellvis) that were coated with $10 \mu \mathrm{g} / \mathrm{mL}$ fibronectin in PBS at $37^{\circ} \mathrm{C}$ for 30 minutes prior to plating. 50nM MitoTracker Deep Red (ThermoFisher) was added for 30 minutes then washed for at least 30 minutes to allow for recovery time before imaging in FluoroBrite (ThermoFisher) media.

Airyscan confocal imaging. Cells were imaged with a $63 \times 1.4$ NA oil objective on a ZEISS 880 LSM Airyscan confocal system with an inverted stage and heated incubation system with $5 \% \mathrm{CO}_{2}$ control. The GFP channels were imaged with a $488 \mathrm{~nm}$ laser line at $\sim 500 \mathrm{nW}$ laser power. The mCherry or tagRFP channels were imaged with $561 \mathrm{~nm}$ laser at $\sim 1 \mu \mathrm{W}$ laser power. The MitoTracker Deep Red channel was imaged with $\sim 250 \mathrm{nW}$ laser power. For timelapse imaging, the zoom factor was set between $3 x-6 x$ to increase the frame rate. In all cases, the maximum pixel dwell time ( $\sim 0.684 \mu \mathrm{s} /$ pixel) and $2 x$ Nyquist optimal pixel size ( $40 \mathrm{~nm} /$ pixel) was used.

Cytoskeleton Stabilized Membrane Extraction of Cells for Fluorescence Imaging. Cells were cultured and imaged as described above. Medium was replaced with cytoskeleton stabilization membrane extraction buffer $(0.05 \%$ saponin, 100mM PIPES, pH 6.8, $1 \mathrm{mM} \mathrm{EGTA,} 1 \mathrm{mM} \mathrm{MgCl}$, 4.2\% sucrose, $1 \mathrm{mM}$ PMSF, $10 \mu \mathrm{M}$ taxol, 10 $\mu \mathrm{M}$ phalloidin-Alexa568) prewarmed to $37^{\circ} \mathrm{C}$. Cells were imaged immediately before and $10 \mathrm{~min}$ after the addition of membrane extraction buffer. 
Antibodies. We used the rabbit anti-Fis1 antibody against the N-terminus cytoplasmic facing side of the human Fis1 protein, made by Prestige Antibodies Powered by Atlas Antibodies (Sigma-Aldrich, catalog \#: HPA017430). The amino acid sequence of the antigen is MEAVLNELVSVEDLLKFEKKFQSEKAAGSVSKSTQFEYAWCLVRSKYNDDIRKGIVLLEELLPKGS KEEQRDYVFYLAVGNYRLKEYEKALKYVRGLLQTEPQNNQAKELERLIDKAMKKD.

Immunofluorescence. Cells were washed in PBS then fixed with 4\% PFA for 30 minutes before permeabilization with $0.1 \%$ Triton X-100 for 30 minutes. Cells were then blocked overnight with $4 \%$ BSA at $4{ }^{\circ} \mathrm{C}$. Cells were then incubated with primary antibody for 2 hours, rinsed $3 x$ with PBS for 10 minutes each, then incubated with secondary antibodies (Jackson Immunoresearch Laboratories) for 1 hour, rinsed 3x with PBS for 10 minutes each, then counterstained with Alexa405-phalloidin (ThermoFisher) for 30 minutes, then rinsed with PBS $3 \times$ for 10 minutes each, then mounted with ProLong Glass antifade reagent (ThermoFisher).

Scanning Electron Microscopy of Membrane Extracted Cells. Cells were briefly washed with $0.1 \mathrm{M}$ phosphate-buffered saline $(\mathrm{PBS})\left(37^{\circ} \mathrm{C}\right)$ to remove culture media and immediately treated with a membrane extraction solution containing 1\% Triton X-100, $100 \mathrm{mM}$ PIPES (pH 7.2), 1 mM EGTA, $1 \mathrm{mM} \mathrm{MgCl2,} \mathrm{4.2 \%} \mathrm{sucrose,} 10 \mu \mathrm{M}$ taxol (Thermo-Scientific) and $10 \mu \mathrm{M}$ phalloidin (Sigma) for $10 \mathrm{~min}$ with gentle rocking at room temperature. Then the samples were washed twice for 5 min in PBS and fixed with 2\% glutaraldehyde, 2\% paraformaldehyde (Electron Microscopy Sciences - EMS), in $0.1 \mathrm{M}$ sodium cacodylate buffer for $30 \mathrm{~min}$ at RT. Samples were washed in the same buffer, post-fixed with $1 \%$ OsO4 (EMS) and $1 \%$ tannic acid (EMS) for $1 \mathrm{~h}$ each, dehydrated with a graded ethanol series until absolute and critical point-dried (Leica CPD 030). The samples were coated with a thin platinum layer (4 $\mathrm{nm}$ ) (Leica EM SCD500) and imaged on a Zeiss Sigma-VP scanning electron microscope at $5 \mathrm{kV}$.

Image processing and analysis. After acquisition, images were Airyscan processed using the auto-filter 2D-SR settings in Zen Blue (ZEISS). All images were post-processed and analyzed using Imaris (BITPLANE) and Fiji software ${ }^{28}$.

Plasmids. Drp1-mCherry was a kind gift from Gia Voeltz (Addgene plasmid \#49152). mCherry-Cytob ${ }_{5} R R$ was a gift from Nica Borgese ${ }^{29}$. EGFP-LifeAct was a gift from Jennifer Rohn ${ }^{30}$. Lamp1-mCherry, SiT-mApple, Sec61-mCherry, and dsRedSkl were all generous gifts from the Lippincott-Schwartz lab. Rab5a-mCherry was a generous gift from the Merrifield lab (Addgene plasmid \#27679). All custom actin nanobody probes were generated starting from the commercial vector of actin chromobody-tagGFP or actin chromobody-tagRFP (ChromoTek) and cloned via the Bglll and Notl restriction sites. The following amino acid sequences were attached to the C-terminal of the actin chromobody probes to target the protein either to mitochondria or the ER:

Fis1 (AC-mito and LifeAct-GFP-Fis1):

IQKETLKGVVVAGGVLAGAVAVASFFLRNKRR ${ }^{5}$

Cytb5mito (aka "CytobsR") (AC-GFP-Cytb5mito and LifeAct-GFP-Cytb5mito):

FEPSETLITTVESNSSWWTNWVIPAISALVVALMYRR ${ }^{31}$

Cytb5ER (AC-ER):

IDSSSSWWTNWVIPAISAVAVALMYRLYMAED ${ }^{5}$

LifeAct-GFP-Fis1, LifeAct-GFP-Cytb5mito, and AC-GFP-Cytb5mito were generated using PFU Ultra II for megaprimer PCR insertion $^{32}$. The PCR primers, intended modifications, insert templates, and destination plasmids are listed in Supplemental Table 1 below. All constructs were sequenced completely across their coding region.

\begin{tabular}{|c|c|c|c|c|}
\hline Insert plasmid & $\begin{array}{l}\text { Destination } \\
\text { plasmid }\end{array}$ & Modification to Destination plasmid & Final Plasmid & Primer Sequence \\
\hline & & $\begin{array}{l}\text { Fis1p localization signal was inserted at the C- } \\
\text { terminus of tagGFP via } 4 \text { rounds of overlapping }\end{array}$ & & $\begin{array}{l}\text { fwd: GCGCGCAGATCTAATGGCTCAGGTGCAGCTGGT } \\
\text { GGA; } \\
\text { rev: ATATAT AGCGGCCGCCTAGCGGCGTTTGTTGCG } \\
\text { CAGAAAAAAGCTCGCCACCGCCACCGCGCCCGCCAGC }\end{array}$ \\
\hline pAC-tagGFP & $\begin{array}{l}\text { pAC-tagGFP- } \\
\text { Fis1p }\end{array}$ & $\begin{array}{l}\text { PCR, G and P were used as a linker in between Fis } 1 \\
\text { and GFP }\end{array}$ & AC-mito (CMV) & $\begin{array}{l}\text { ACGCCGCCCGCCACCACCACGCCTTTCAGGGTTTCTTT } \\
\text { CTGAATCGGCCCCCTGTACAGCTCGTC }\end{array}$ \\
\hline
\end{tabular}


bioRxiv preprint doi: https://doi.org/10.1101/639278; this version posted June 25, 2019. The copyright holder for this preprint (which was not certified by peer review) is the author/funder, who has granted bioRxiv a license to display the preprint in perpetuity. It is made available under aCC-BY-NC-ND 4.0 International license.

\begin{tabular}{|c|c|c|c|c|}
\hline pmCherry & mCherry-Fis1p & $\begin{array}{l}\text { Fis1p localization signal was inserted at the C- } \\
\text { terminus of mCherry via } 4 \text { rounds of overlapping } \\
\text { PCR, G and P were used as a linker in between Fis1 } \\
\text { and mCherry }\end{array}$ & mCherry-mito & $\begin{array}{l}\text { GGAG; } \\
\text { rev: ATATATAGCGGCCGCCTAGCGGCGTTTGTTGCGC } \\
\text { AGAAAAAAGCTCGCCACCGCCACCGCGCCCGCCAGCA } \\
\text { CGCCGCCCGCCACCACCACGCCTTTCAGGGTTTCTTTC } \\
\text { TGAATCGGCCCCCTGTACAGCTCGTC }\end{array}$ \\
\hline pAC-tagGFP & AC-ER & $\begin{array}{l}\text { Cytb5ER localization signal was inserted at the C- } \\
\text { terminus of tagGFP via } 4 \text { rounds of overlapping } \\
\text { PCR, G and P were used as a linker in between } \\
\text { Cytb5ER and tagGFP }\end{array}$ & $\begin{array}{l}\text { AC-ER (CMV } \\
\text { promoter) }\end{array}$ & $\begin{array}{l}\text { fwd: GCGCGCAGATCTAATGGCTCAGGTGCAGCTGGTG } \\
\text { GA; } \\
\text { rev: ATATATAGCGGCCGC CTAATCTTCCGCCATATACA } \\
\text { GGCGATACATCAGCGCCACCGCCACCGCGCTAATCGCC } \\
\text { GGAATCACCCAGTTGGTCCACCAGCTGCTGCTGCTATC } \\
\text { AAT CGGCCCCCTGTACAGCTCGTC }\end{array}$ \\
\hline $\begin{array}{l}\text { mcherry- } \\
\text { Cyto } b_{5} \text { RR }\end{array}$ & EGFP-Lifeact & $\begin{array}{l}\text { insertion of CytoB5RR sequence at C-terminus of } \\
\text { EGFP with GGSGGGGS linker in between }\end{array}$ & $\begin{array}{l}\text { LifeAct-GFP- } \\
\text { Cytb5mito }\end{array}$ & $\begin{array}{l}\text { fwd: TCTCGGCATGGACGAGCTGTACAAGGGTGGATC } \\
\text { TGGAGGTGGCG } \\
\text { rev: GATTATGATCTAGAGTCGCGGCCGCTTATTAACG } \\
\text { CCGGTACATCAGCGCTA }\end{array}$ \\
\hline $\begin{array}{l}\text { pAC-tagGFP- } \\
\text { Fis1p }\end{array}$ & EGFP-Lifeact & insertion of Fis1p sequence at C-terminus of EGFP & LifeAct-GFP-Fis1 & $\begin{array}{l}\text { fwd: TCTCGGCATGGACGAGCTGTACAAGGGTGGATC } \\
\text { TGGAGGTGGCG } \\
\text { rev:TCTCGGCATGGACGAGCTGTACAAGGGGCCGATT } \\
\text { CAGAAAGAAACCCT }\end{array}$ \\
\hline $\begin{array}{l}\text { mcherry- } \\
\text { Cyto } b_{5} \text { RR }\end{array}$ & AC-mito & $\begin{array}{l}\text { replace Fis1p localization signal with CytoB5RR } \\
\text { signal with a GGSGGGGS linker in between }\end{array}$ & AC-GFP-Cytb5mito & $\begin{array}{l}\text { fwd: GATTATGATCTAGAGTCGCGGCCGCTTAGCGGC } \\
\text { GTTTGTTGCGCAGAAAAAA } \\
\text { rev: ACGGCATGGACGAGCTGTACAGGGGTGGATCTG } \\
\text { GAGGTGGCG }\end{array}$ \\
\hline $\begin{array}{l}\text { pLV hUbC- } \\
\text { dCas9 VP64- } \\
\text { T2A-GFP }\end{array}$ & $\begin{array}{l}\text { pSin-CMV-AC- } \\
\text { mito }\end{array}$ & $\begin{array}{l}\text { The UbC promoter region was synthesized from } \\
\text { pLV hUbC-dCas9 VP64-T2A-GFPusing Q5 Hot Start } \\
\text { High-Fidelity DNA polymerase with additions of } \\
\text { the EcoRI (5'end) and Nhel ( } 3^{\prime} \text { end) restriction } \\
\text { enzyme sites. The DNA fragment of Ac-TagGFP- } \\
\text { Fis1 DNA fragment was taken from pSin-CMV-AC- } \\
\text { TagGFP- Fis1 by cutting with Nhel and KpnI } \\
\text { restriction enzymes. Two DNA fragments were } \\
\text { cloned into the pSin lentiviral vector digested with } \\
\text { EcoRI and Kpnl. }\end{array}$ & $\begin{array}{l}\text { AC-mito } \\
\text { (UbC promoter- } \\
\text { used in } \\
\text { experiments) }\end{array}$ & $\begin{array}{l}\text { fwd: TTAAGGGAATTCGGCCTCCGCGCCG; } \\
\text { rev: GCCCAAGCTAGCGTCTAACAAAAAAG } \\
\text { CCAAAAACGG. }\end{array}$ \\
\hline $\begin{array}{l}\text { pLV hUbC- } \\
\text { dCas9 VP64- } \\
\text { T2A-GFP }\end{array}$ & $\begin{array}{l}\text { pSin-CMV-AC- } \\
\text { ER }\end{array}$ & $\begin{array}{l}\text { The UbC promoter region was synthesized from } \\
\text { pLV hUbC-dCas9 VP64-T2A-GFP with additions of } \\
\text { the EcoRI (5'end) and Nhel ( } 3 \text { 'end) restriction } \\
\text { enzyme sites. The DNA fragment of Ac-TagGFP- } \\
\text { Cytb5 DNA fragment was taken from pSin-CMV- } \\
\text { AC-TagGFP-Cytb5 by cutting with Nhel and Kpnl } \\
\text { restriction enzymes. The two DNA fragments were } \\
\text { cloned into the pSin lentiviral vector digested with } \\
\text { EcoRI and Kpnl. }\end{array}$ & $\begin{array}{l}\text { AC-ER } \\
\text { (UbC promoter - } \\
\text { used in } \\
\text { experiments) }\end{array}$ & $\begin{array}{l}\text { fwd: TTAAGGGAATTCGGCCTCCGCGCCG; } \\
\text { rev: GCCCAAGCTAGCGTCTAACAAAAAAG } \\
\text { CCAAAAACGG. }\end{array}$ \\
\hline $\begin{array}{l}\text { pAC-TagGFP- } \\
\text { Cytb5 }\end{array}$ & mCherry-mito & $\begin{array}{l}\text { pmCherry-Fis1 plasmid was cut with BsrGI and } \\
\text { Notl restriction enzymes to remove the Fis1 } \\
\text { coding region and replaced with the Cytb5 coding } \\
\text { region cut with BsrGl and Notl restriction enzymes } \\
\text { from the pAC-TagGFP-Cytb5 plasmid using Quick } \\
\text { Ligation Kit. }\end{array}$ & ER-mCherry & n/a. \\
\hline
\end{tabular}




\section{Acknowledgements}

Many thanks to Andrew Moore (Janelia Farms) and Stephanie Harada (Salk Institute for Biological Studies) for help with the cartoon diagram for Figure 1. We would like to also thank Christopher Obara and Jennifer Lippincott-Schwartz (Janelia Farms) for critical feedback and suggestions on the manuscript. This work was also greatly improved by Twitter feedback on our initial bioRxiv preprint, most especially Subhojit Roy (UW-Madison). The Waitt Advanced Biophotonics Center is funded by the Waitt Foundation and Core Grant applications NCI CCSG (CA014195) and NINDS Neuroscience Center (NS072031). This work was supported by the Transgenic Core Facility of the Salk Institute with funding from NIHNCI CCSG: P30 014195. R.G. laboratory is funded by grants from HFSP RGP0021/2016 and the Cluster of Excellence CIBSS- Centre for Integrative Biological Signalling Studies. O.A.Q. lab is supported by NIGMS Grant R15 GM119077 and by funding from the University of Richmond School of Arts \& Sciences. 
a

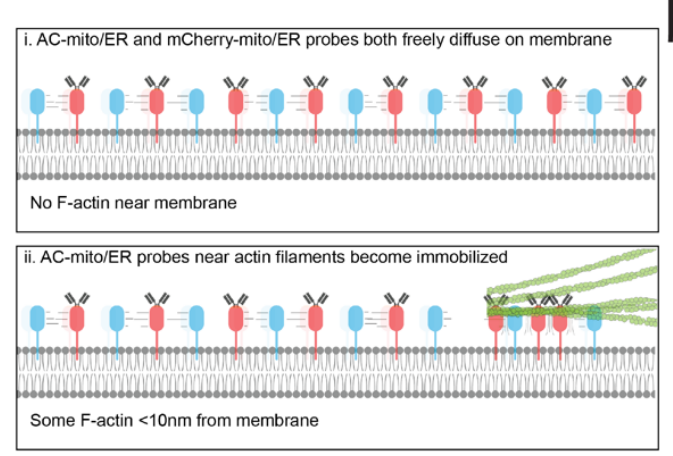

iii. More actin filaments beget more AC-mito/ER probe immobilization

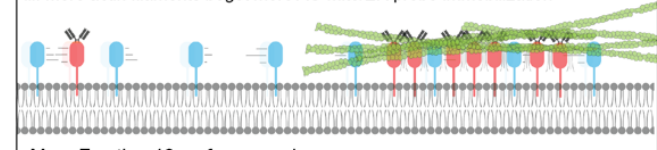

More F-actin <10nm from membrane

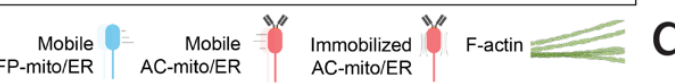

C
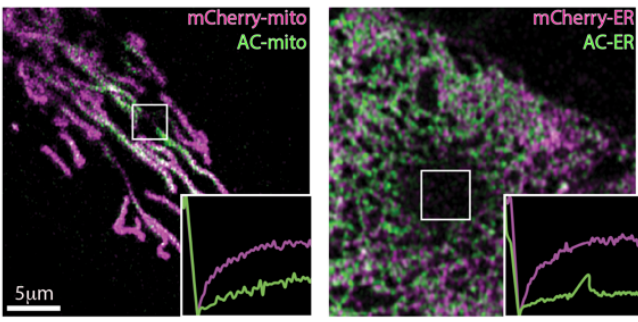

\begin{tabular}{|c|c|c|}
\hline & Immobile Fraction & $\mathbf{t}_{1 / 2}(\mathrm{sec})$ \\
\hline mCherry-mito & $0.40 \pm 0.06$ & $6.14 \pm 1.033$ \\
\hline AC-mito & $0.58 \pm 0.06$ & $8.53 \pm 2.379$ \\
\hline mCherry-ER & $0.30 \pm 0.06$ & $7.68 \pm 0.89$ \\
\hline AC-ER & $0.54 \pm 0.049$ & $10.14 \pm 0.94$ \\
\hline
\end{tabular}

b
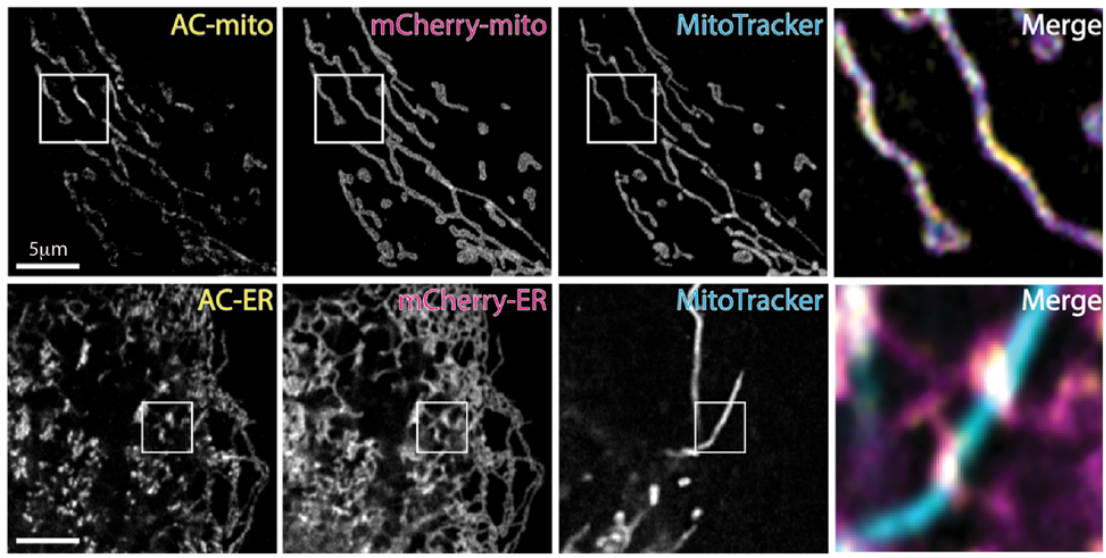

d
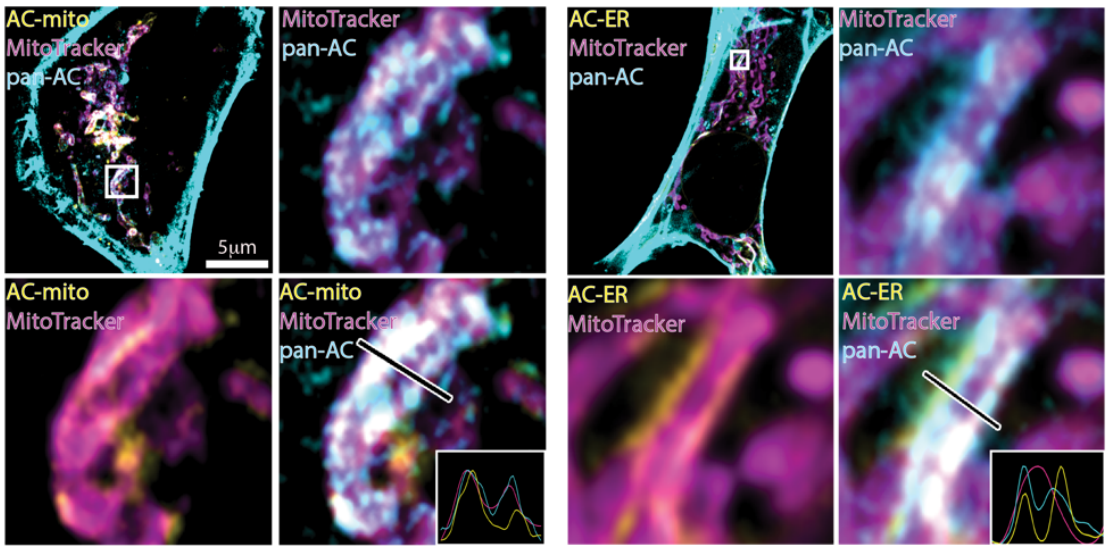

Figure 1|Mitochondria and ER localized actin nanobodies reveal sub-organellar accumulation of actin

filaments. a,Cartoon depicting change in AC-mito/ER localization in response to the presence of actin. When there is no actin near the membrane, AC-mito/ER freely diffuse throughout the membrane. When actin comes within $10 \mathrm{~nm}$ of the membrane, only the AC probes are immobilized at the sites of actin accumulation. As actin filaments grow/accumulate, more of the AC probe becomes immobilized. $\mathbf{b}$, Co-expression of mCherrymito/ER in U2OS cells demonstrates AC-mito/ER sub-accumulation depends on actin nanobody activity. Subaccumulation was observable in all cells imaged (AC-mito: $n=68, A C-E R: n=55$ ). Scale bars: $5 \mu$ m. C, FRAP of cells co-expressing AC/mCherry- mito/ER shows that both probes are mobile, but the AC probes have lower mobility and a higher immobile fraction. $\mathbf{d}$, Co-expression of a pan-actin label (ACtagRFP) with AC-mito/ER reveals actin is enriched at sites labeled by AC-mito/ER probes. 

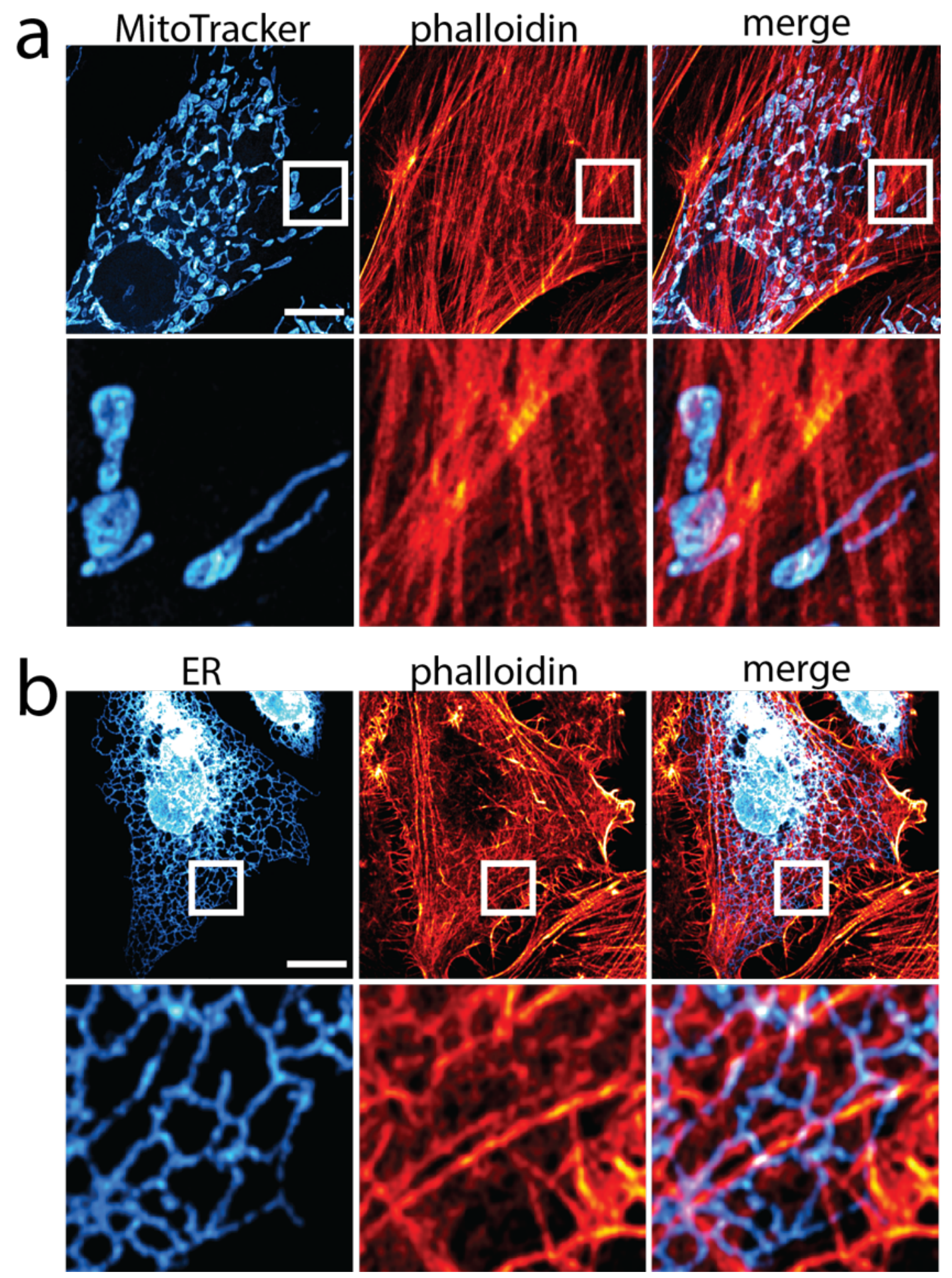

Supplementary Figure 1|Phalloidin staining does not show obvious accumulation of actin on mitochondria or ER. a, Cells stained with phalloidin and MitoTracker do not show obvious accumulation of actin on mitochondria. $\mathbf{b}$, Cells expressing the ER marker Sec61-mCherry counterstained with phalloidin do not show obvious accumulation of actin on any specific region of the ER. Scale bar: $10 \mu \mathrm{m}$. 
a

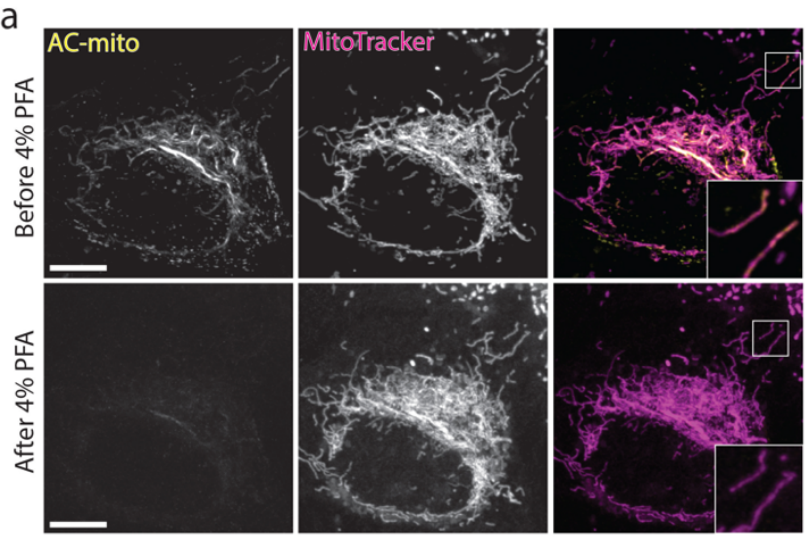

b
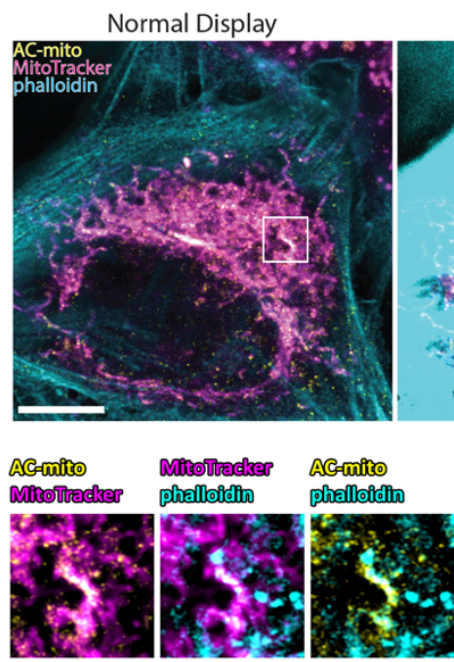

Adjusted Contrast

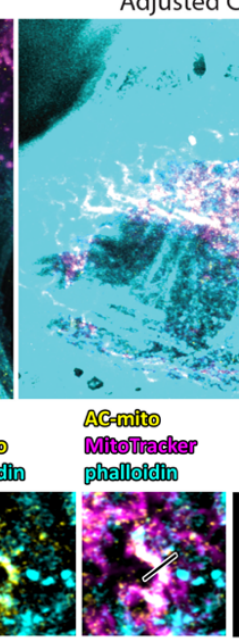

C
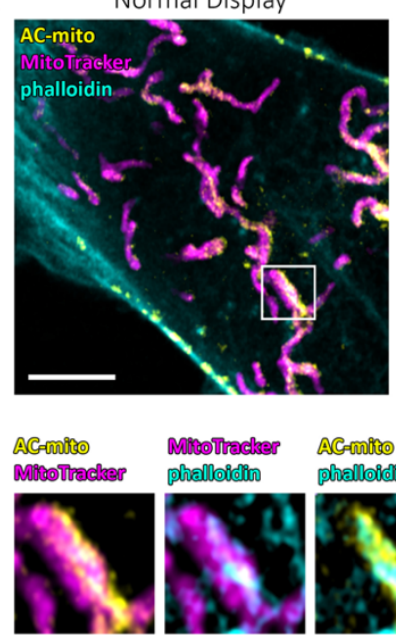

Enhanced Contrast

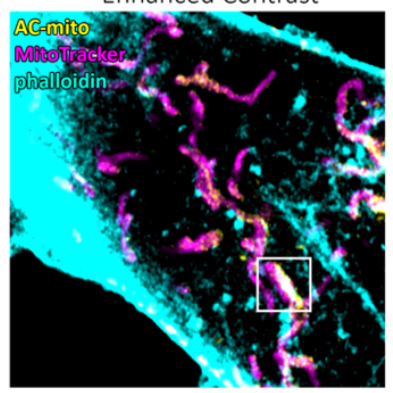

Acmoriso Merotracker phalloidion

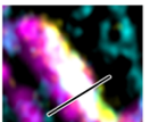

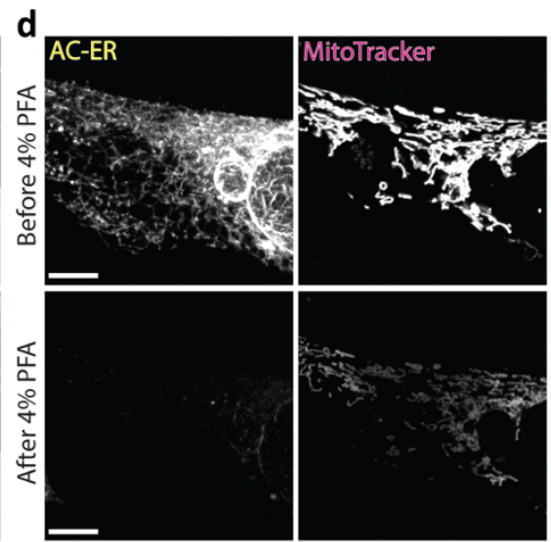
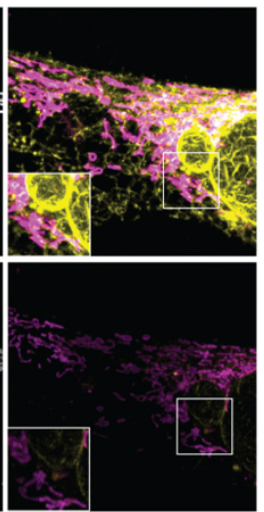

e

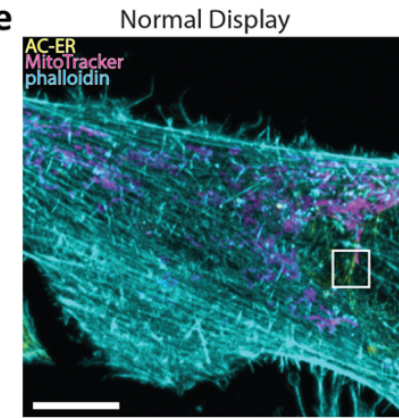

Adjusted Contrast

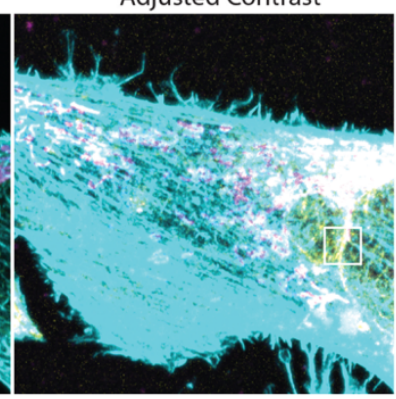

Acrantio

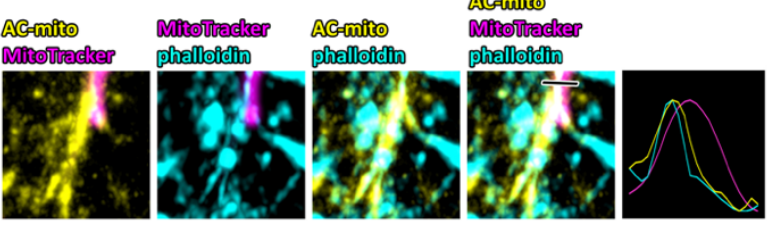

f

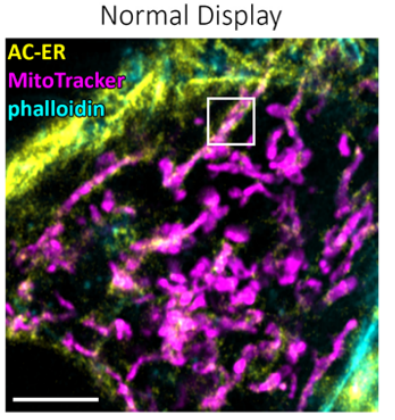

ACESR Mocotracke

ACEER

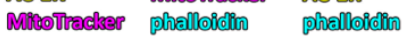

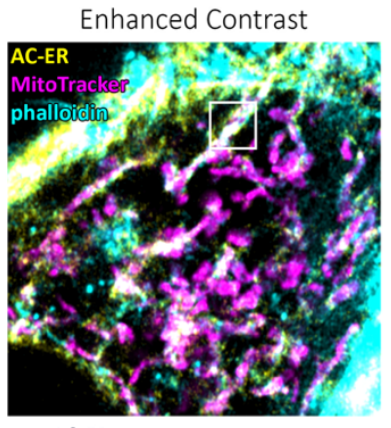

ACFER

Mreolliacker phealloridion
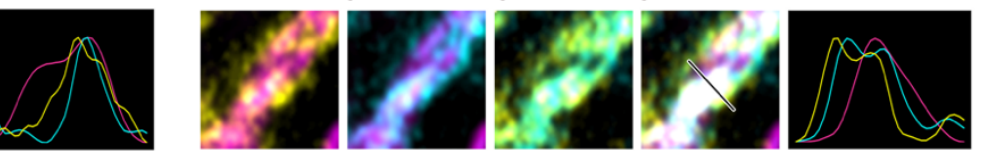

Supplementary Figure 2|Most sub-organellar actin accumulation does not survive conventional fixation. $\mathbf{a + d}$ Imaging the same cell expressing AC-mito or AC-ER before and 30 minutes after fixation with $4 \%$ PFA reveals a significant decrease in AC-mito or AC-ER signal after fixation. This decrease was observed in all cells imaged (AC-mito: $n=3, A C$-ER: $n=4$ ). $b+e$, phalloidin colocalizes with AC-mito and AC-ER, but with much lower signal than surrounding larger actin structures. Bottom row shows insets with adjusted contrast. $\mathbf{c}+\mathbf{f}$, Cells permeablized with cytoskeleton stabilizing membrane extraction to label phalloidin while preserving ACmito and AC-ER signal. Phalloidin colocalizes with AC-mito and AC-ER. Scale bars: $10 \mu \mathrm{m}$ 

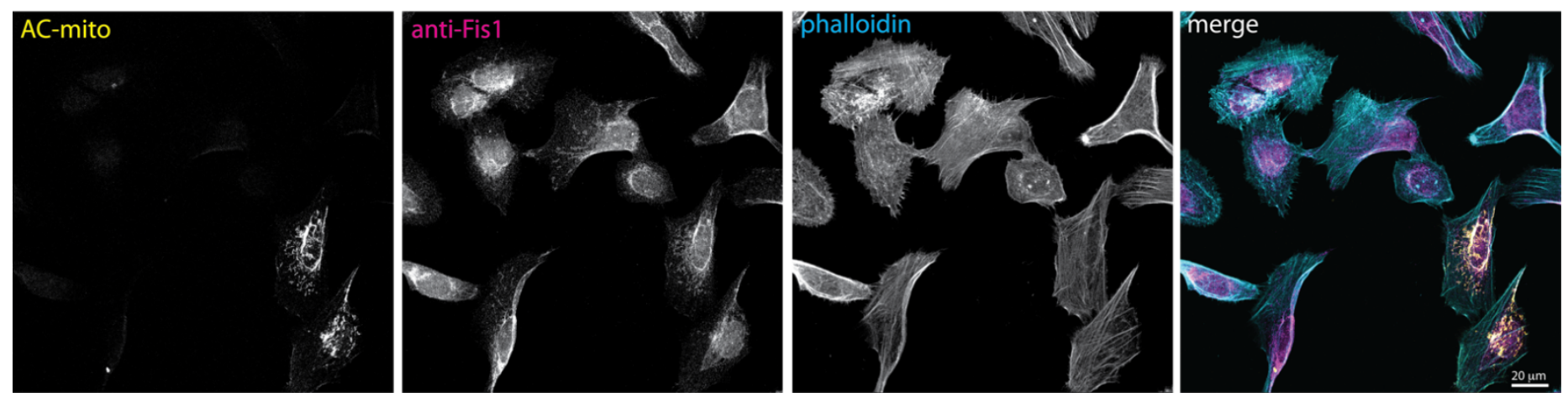

Supplementary Figure 3|AC-mito expression does not alter endogenous Fis1 localization to mitochondria. Cells expressing AC-mito show similar levels of endogenous anti-Fis1 immunofluorescence as non-transfected cells. Note that the anti-Fis1 antigen is not present in the AC-mito protein. Scale bar: $20 \mu \mathrm{m}$. 

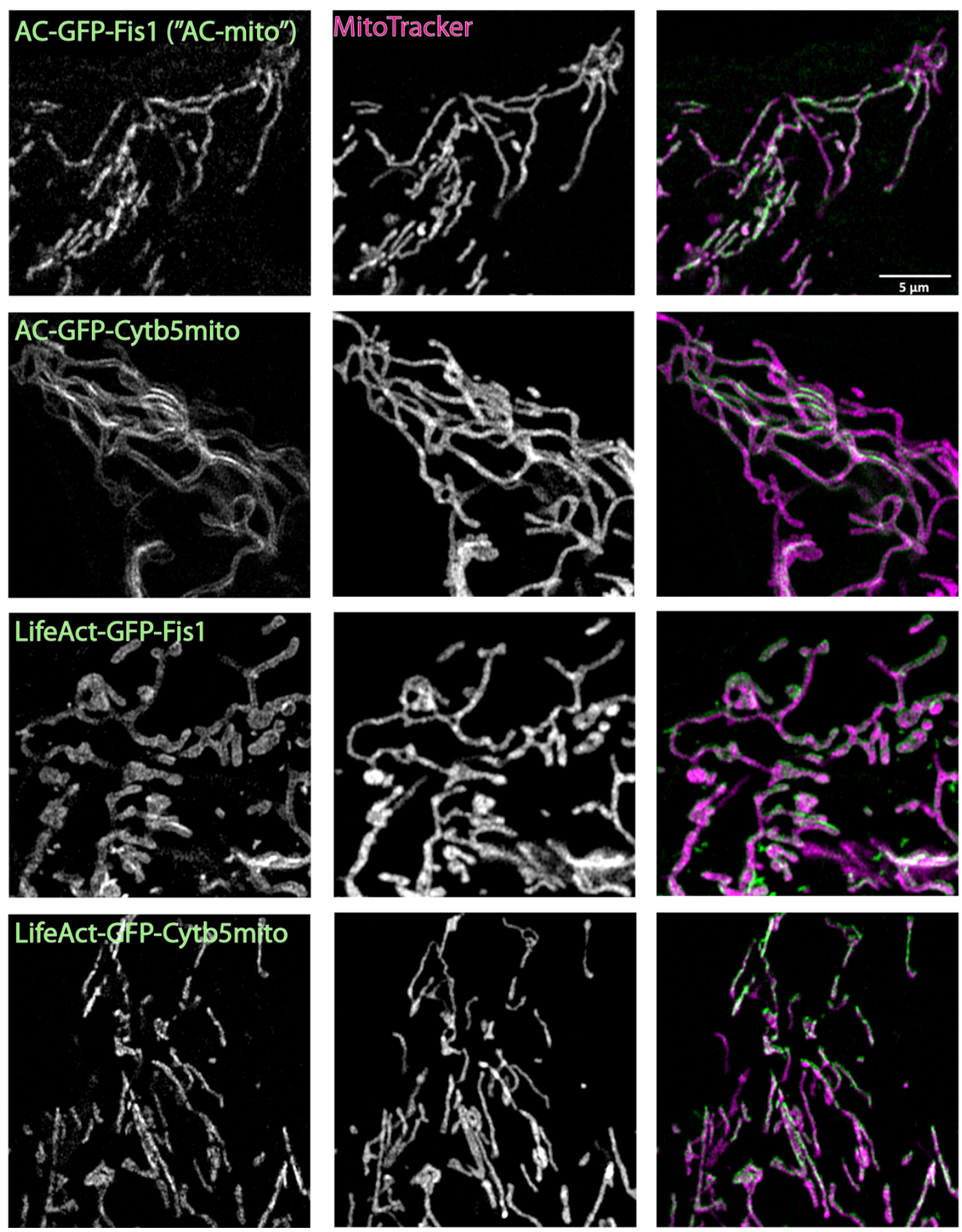

Supplementary Figure 4|Alternate membrane-targeting and actin-binding motifs yield similar results. Switching the Fis1 mitochondrial outer membrane targeting sequence for Cytb5mito (Cytb5mito-AC-GFP) yields similar results. Similarly, switching the actin nanobody motif with LifeAct (Fis1-LifeAct-GFP) also yields similar results. Scale bar: $5 \mu \mathrm{m}$. 


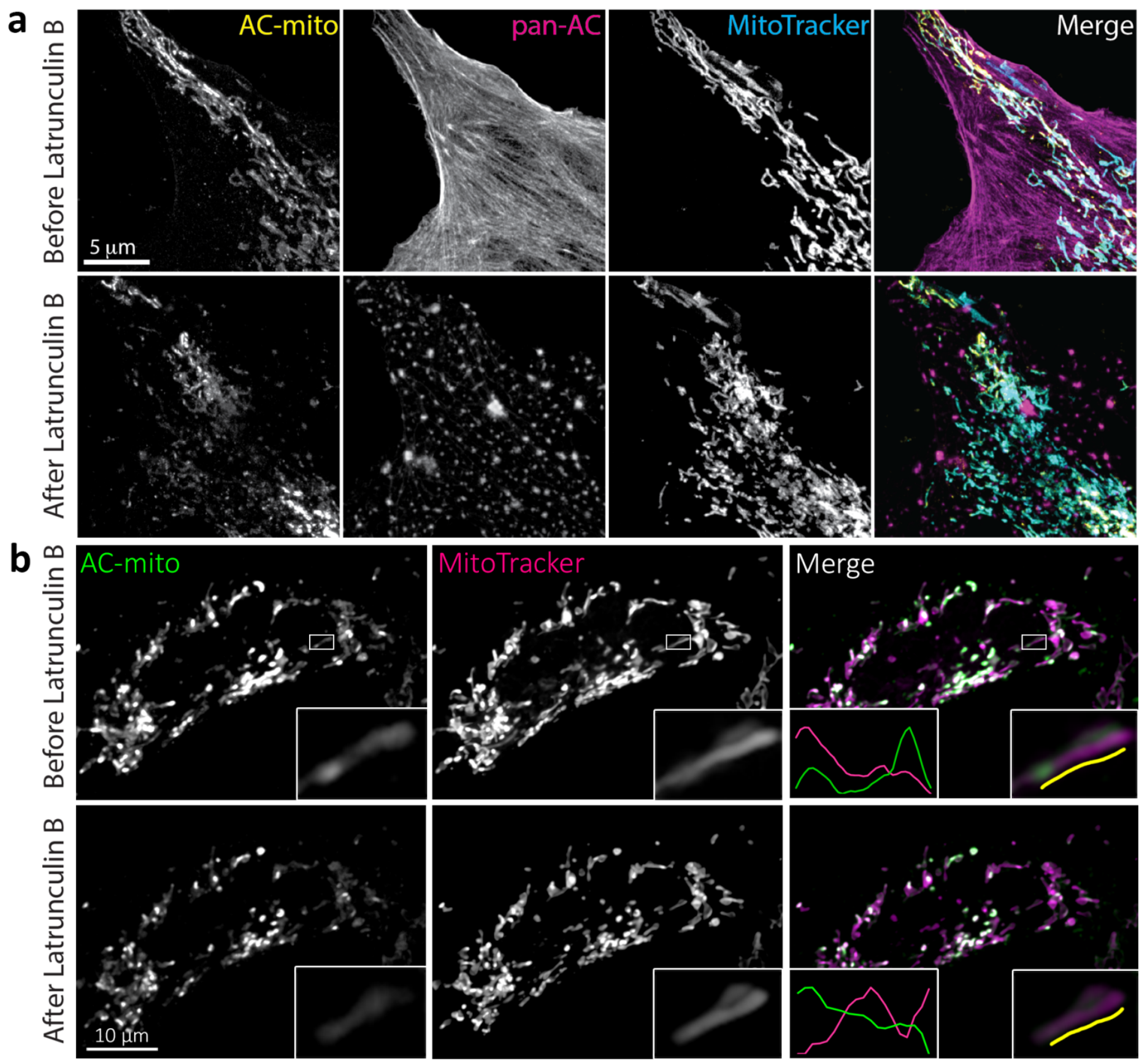

Supplementary Figure 5|Actin depolymerization destroys AC-mito localization. a,Cells expressing AC-mito and pan-AC (ACtagRFP) were imaged before and after treatment with Latrunculin $B$ for 30 minutes. After Latrunculin B treatment, AC-mito displayed much less accumulation at specific regions on mitochondria. This loss of accumulation was observed in all cells imaged after treatment with Latrunculin B or Cytochalasin D ( $n=$ 6). Scale bar: $5 \mu \mathrm{m} \mathrm{b}$, Cells expressing AC-mito before and after treatment with Latrunculin B including example insets. The more diffuse localization of AC-mito following Latrunculin B treatment is apparent in the example mitochondrion. Scale bar: $10 \mu \mathrm{m}$ 

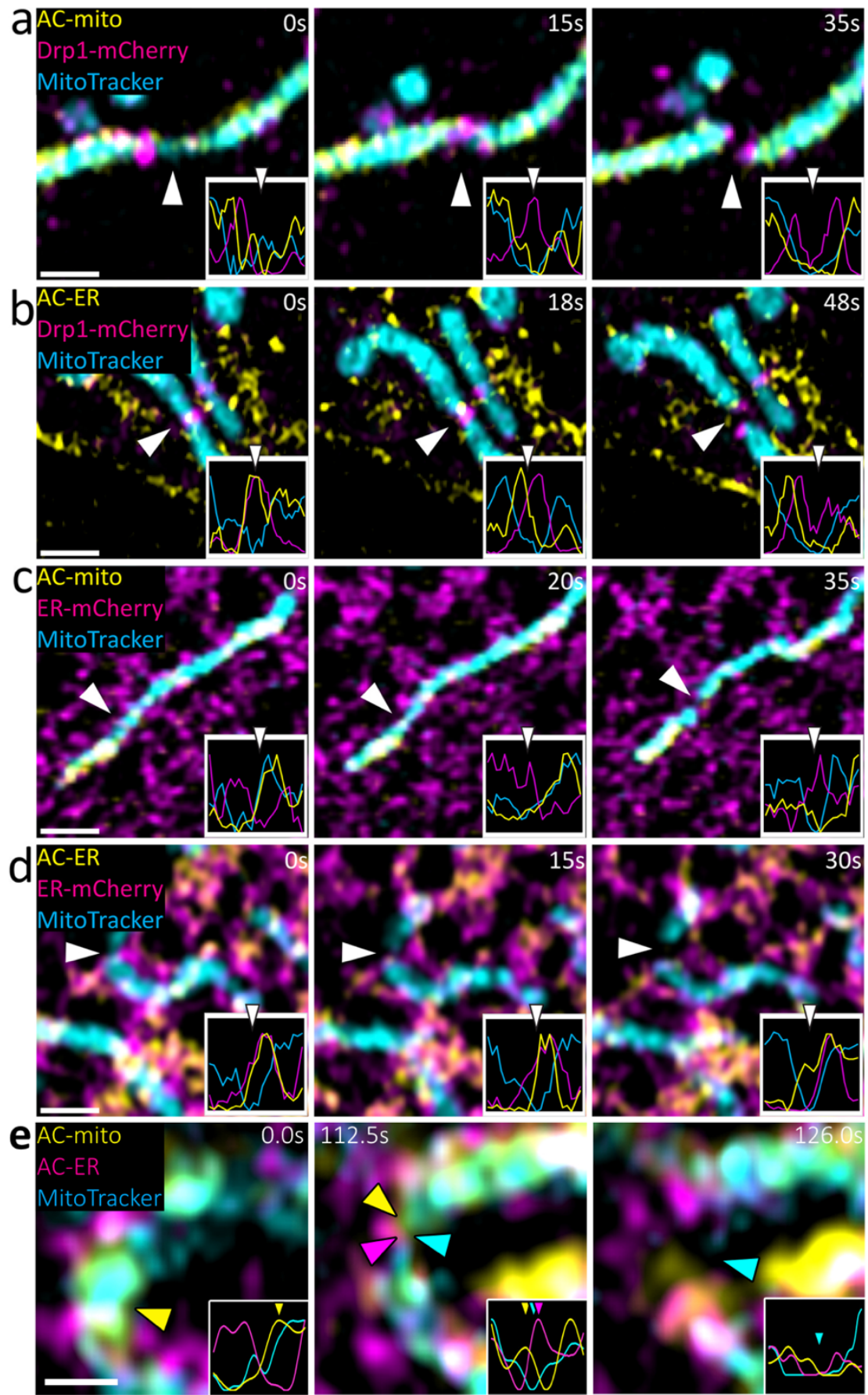

Figure 2| Mitochondria- and ER-associated actin accumulates during mitochondria fission. a, AC-mito accumulates at mitochondrial fission sites prior to Drp1. b, AC-ER co-accumulates with Drp1 during mitochondrial fission. c, AC-mito accumulates at ER-mediated mitochondrial fission sites. d, AC-ER accumulates at ER-tubules driving mitochondrial fission. Scale bars: $1 \mu \mathrm{m}$. e, AC-mito accumulates at mitochondrial fission sites prior to AC-ER accumulation. Scale bar: $0.5 \mu \mathrm{m}$. In total, AC-mito and AC-ER accumulation was observed at nearly all mitochondrial fission events (AC-mito: 32/33 fission events, AC-ER: 31/31 fission events). 

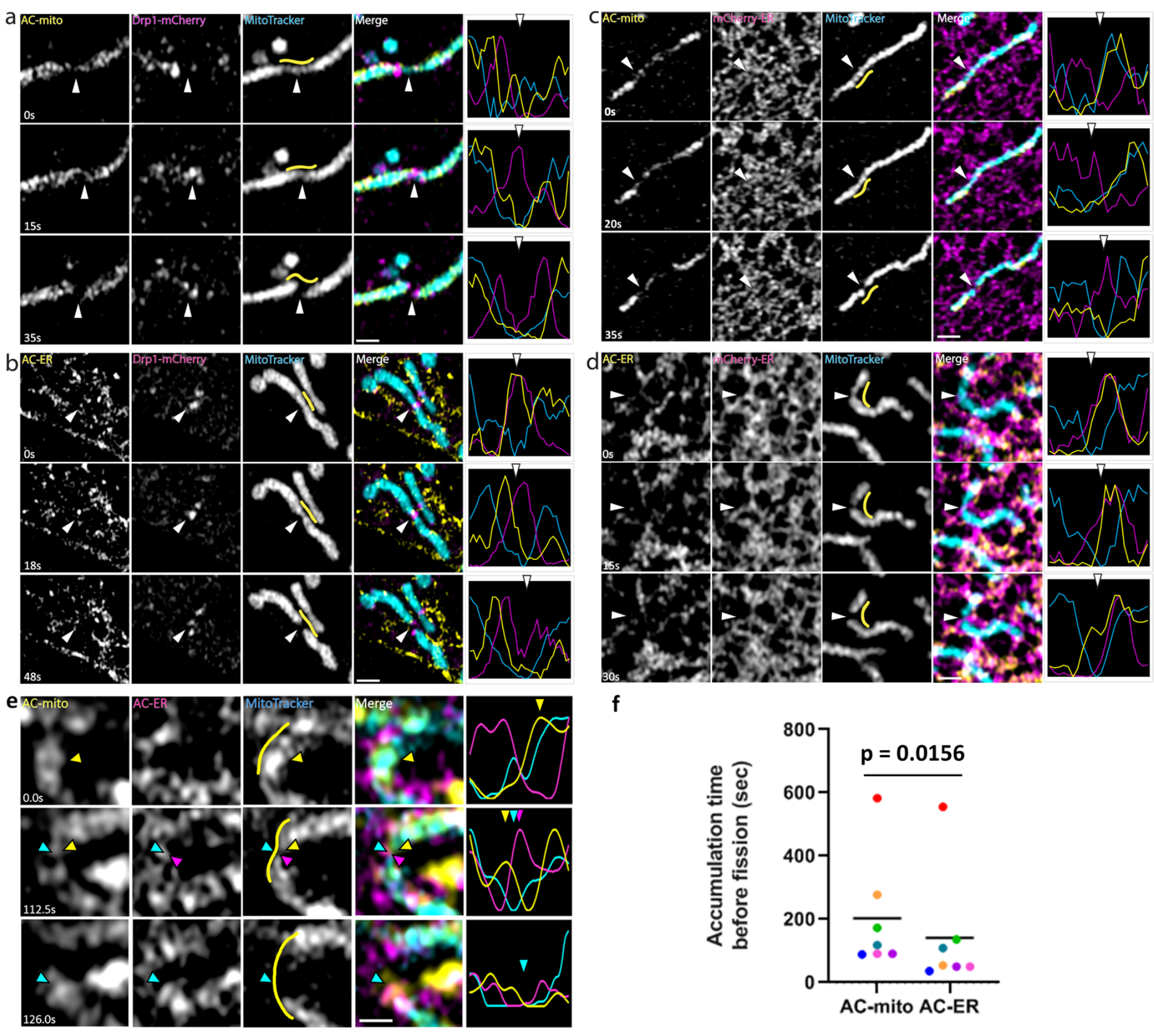

Supplementary Figure 6|Live imaging of AC probes reveal actin accumulation at mitochondrial fission sites. a, AC-mito accumulates prior to Drp1 during mitochondrial fission. $\mathbf{b}$, AC-ER accumulates at mitochondrial fission sites with Drp1. c, AC-mito accumulates at ER-mitochondria contact sites prior to mitochondrial fission. d, AC-ER specifically accumulates at ER-mitochondria contact sites prior to mitochondrial fission. e, AC-mito accumulates at mitochondrial fission sites prior to AC-ER accumulation. Scale bars: $1 \mu \mathrm{m}$. f, Quantification of e. $\mathrm{p}$-value determined by paired ratio t-test. 

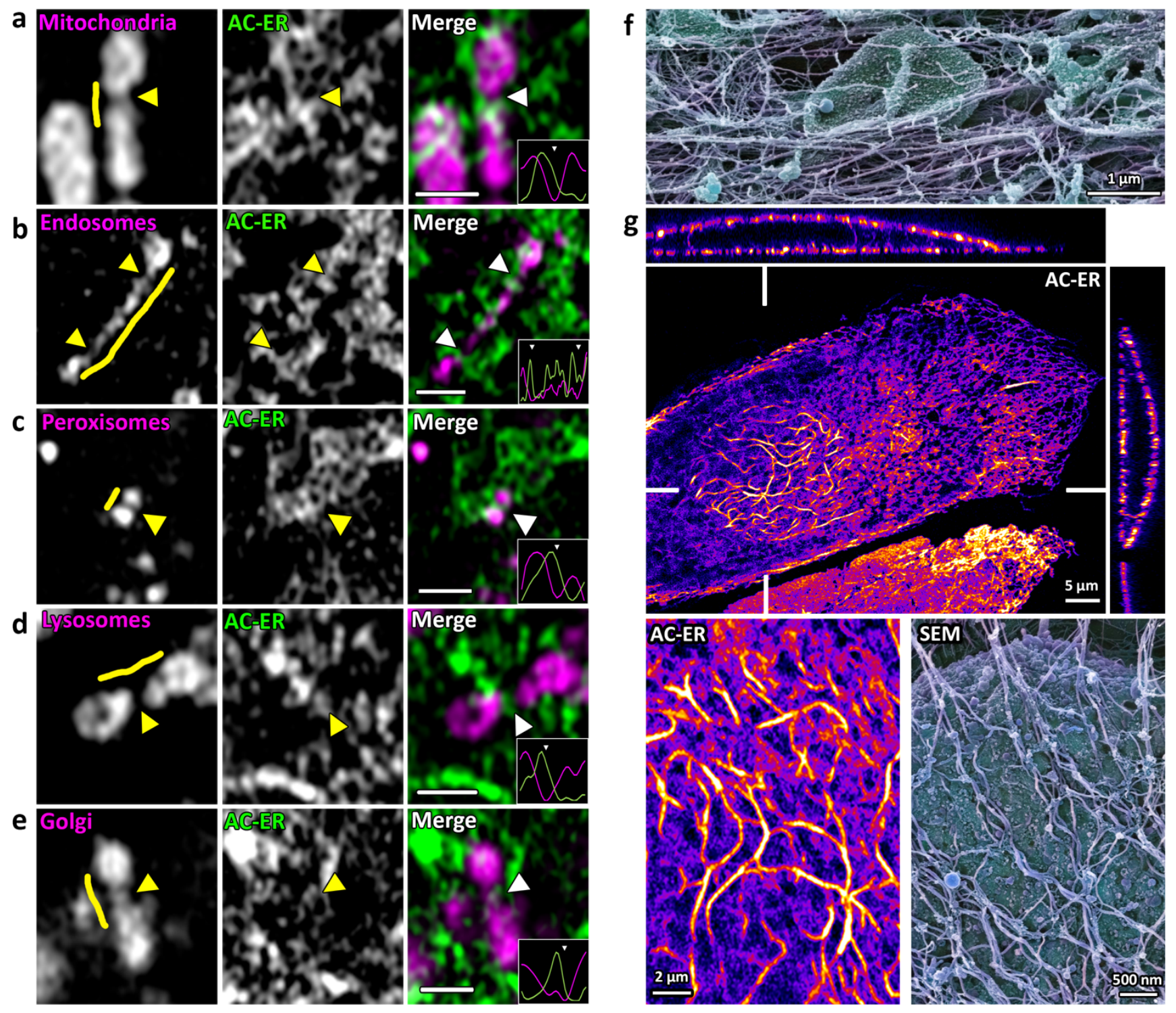

Figure 3|ER-associated actin accumulates at most organelle fission sites. AC-ER accumulates at fission sites in (a) mitochondria; (b) endosomes (labeled with Rab5a-mCherry); (c) peroxisomes (labeled with dsRed-Skl); (d) lysosomes (labeled with LAMP1-mCherry); and (e) Golgi (labeled with SiT-mApple). AC-ER accumulation was observable at nearly all fission/constriction events imaged (mitochondria: 31/31, endosomes: 17/17, peroxisomes: $12 / 12$, lysosomes: $21 / 22$, Golgi: 6/6. (f) SEM image of actin filament association with a mitochondrion. (g) AC-ER reveals a distinct pattern of ER-associated actin accumulation around the nucleus. This pattern was observed in 16/22 cells expressing AC-ER. SEM imaging of membrane extracted cells reveals similar structures. Scale bars for a-e: $1 \mu \mathrm{m}$ 

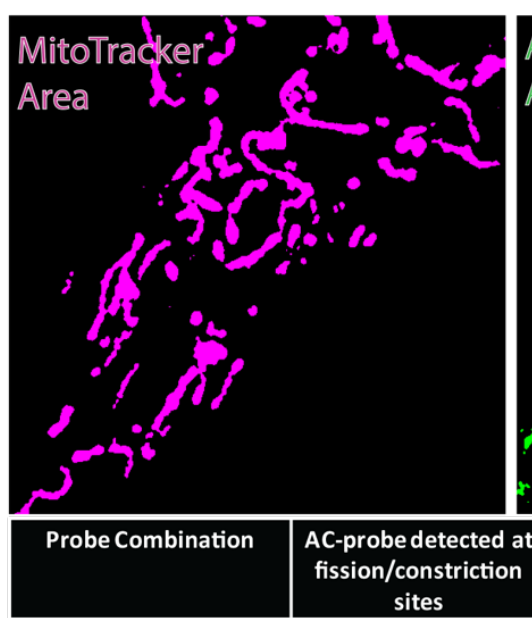

AC-mito + Mitochondria

AC-ER + Mitochondria

AC-ER + Endosomes

AC-ER + Peroxisomes

AC-ER + Lysosomes

$\mathrm{AC}-\mathrm{ER}+\mathrm{Golgi}$

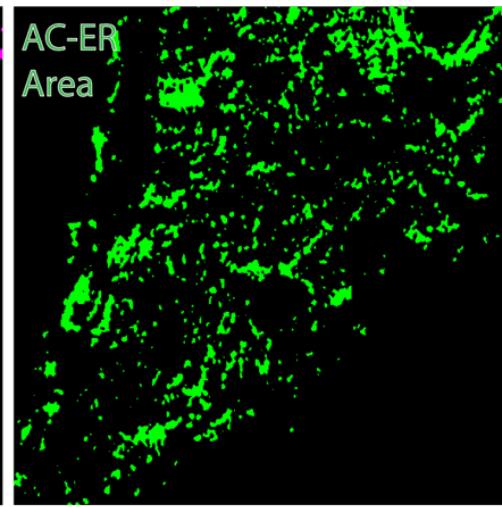

\section{Expected $\mathrm{N}$ of \\ fission/constriction with AC-probe if due to chance}

\begin{tabular}{|c|c|c|c|}
\hline $32(97 \%)$ & $12(36.2 \%)$ & 33 & $1.3 \times 10^{-7}$ \\
\hline $31(100 \%)$ & $7(22.3 \%)$ & 31 & $5.4 \times 10^{-11}$ \\
\hline $17(100 \%)$ & $<1(4.1 \%)$ & 17 & $1.5 \times 10^{-8}$ \\
\hline $12(100 \%)$ & $2(18.3 \%)$ & 12 & 0.00034 \\
\hline $21(95.5 \%)$ & $1(6.4 \%)$ & 22 & $5.1 \times 10^{-9}$ \\
\hline $6(100 \%)$ & $<1(3.5 \%)$ & 6 & 0.015 \\
\hline
\end{tabular}

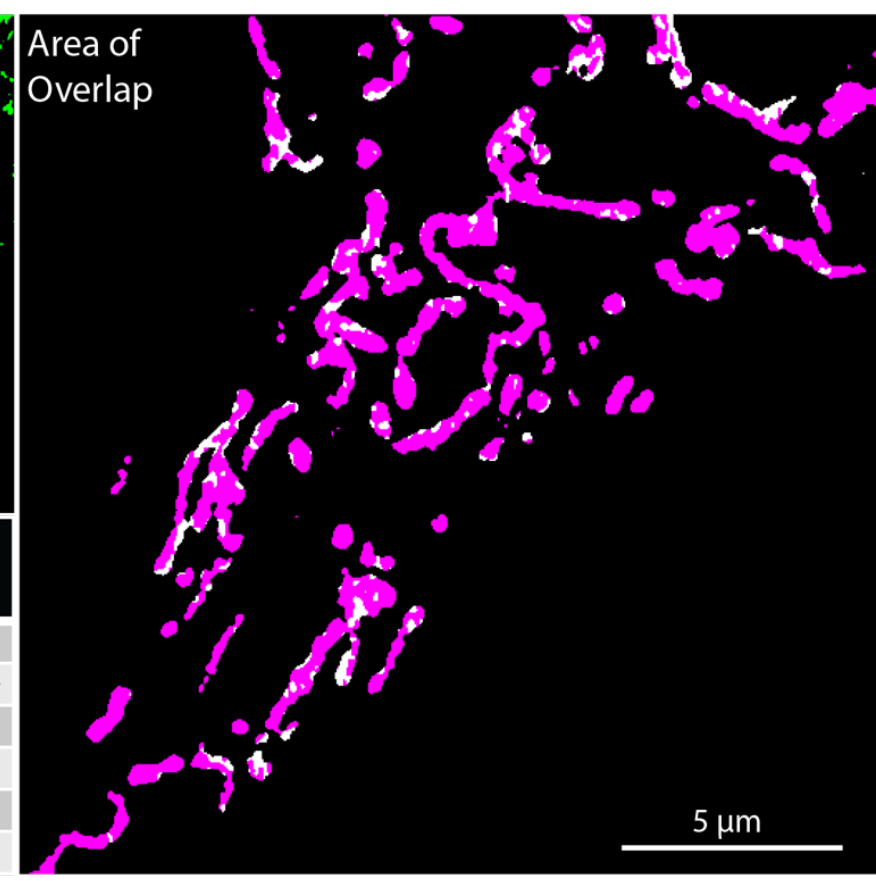

Supplementary Figure 7|AC-mito and AC-ER accumulate at sites of organelle fission/constriction at a significantly higher frequency than would be expected by chance. Images show an example of the masked areas of MitoTracker, AC-ER, and areas of overlap. The table displays the observed frequency of AC probes observed at fission/constriction sites compared to the frequency that would be expected by chance. P-values determined by Fisher's exact test. 
Supplementary Movie 1/Live cell imaging of mitochondria-associated actin. A Hap1 cell expressing AC-mito counterstained with MitoTracker shows dynamic subdomains of actin enrichment on mitochondria.

Supplementary Movie 2/ Live cell imaging of mitochondria-associated actin. A Hap1 cell expressing AC-mito counterstained with MitoTracker shows dynamic subdomains of actin enrichment on mitochondria (example 2).

Supplementary Movie 3|Live cell imaging of ER-associated actin. A Hap1 cell expressing AC-ER counterstained with MitoTracker shows dynamic subdomains of actin enrichment on the ER, in particular at ER-mitochondria contact sites.

Supplementary Movie 4| Live cell imaging of ER-associated actin. A Hap1 cell expressing AC-ER counterstained with MitoTracker shows dynamic subdomains of actin enrichment on the ER, in particular at ER-mitochondria contact sites (example 2).

Supplementary Movie 5|FRAP of cell co-expressing AC-mito and mCherry-mito. FRAP of U2OS cells coexpressing AC-mito and mCherry-mito shows that AC-mito is highly mobile but less mobile than mCherry-mito.

Supplementary Movie 6|FRAP of cell co-expressing AC-ER and mCherry-ER. FRAP of U2OS cells co-expressing AC-ER and mCherry-ER shows that AC-ER is highly mobile but less mobile than mCherry-ER.

Supplementary Movie 7| Live imaging of AC-mito expressing cells before and after Cytochalasin D treatment. Live imaging of Cytochalasin D untreated (left) vs. treated (right) AC-mito expressing U2OS cells.

Supplementary Movie 8| Live imaging of AC-mito and Drp1 during mitochondrial fission. Live imaging of ACmito and Drp1-mCherry in U2OS cells counterstained with MitoTracker reveals accumulation of mitochondriaassociated actin prior to Drp1-mediated fission.

Supplementary Movie 9| Live imaging of AC-ER and Drp1 during mitochondrial fission. Live imaging of AC-ER and Drp1-mCherry in U2OS cells counterstained with MitoTracker reveals accumulation of ER-associated actin prior to Drp1-mediated fission. Scale bar: $1 \mu \mathrm{m}$.

Supplementary Movie 10 | Live imaging of AC-mito and the ER during mitochondrial fission. Live imaging of AC-mito and mCherry-ER in U2OS cells counterstained with MitoTracker reveals accumulation of mitochondria-associated actin prior to ER-mediated fission. Scale bar: $1 \mu \mathrm{m}$.

Supplementary Movie 11 | Live imaging of AC-ER and the ER during mitochondrial fission. Live imaging of ACER and mCherry-ER in U2OS cells counterstained with MitoTracker reveals accumulation of ER-associated actin prior to ER-mediated fission. Scale bar: $1 \mu \mathrm{m}$.

Supplementary Movie 12 |Live imaging of AC-mito and AC-ER during mitochondrial fission. Live imaging of AC-mito and AC-ER in U2OS cells counterstained with MitoTracker reveals accumulation of mitochondriaassociated actin prior to accumulation of ER-associated actin. Scale bar: $1 \mu \mathrm{m}$.

Supplementary Movie 13|ER-associated actin accumulates at mitochondrial fission sites. AC-ER accumulation at a fission site in MitoTracker-labeled mitochondria. Scale bar: $1 \mu \mathrm{m}$ 
Supplementary Movie 14|ER-associated actin accumulates at endosomal fission sites. AC-ER accumulation at two fission sites in Rab5a-mCherry-labeled endosomes. Scale bar: 1 mm

Supplementary Movie 15|ER-associated actin accumulates at peroxisomal fission sites. AC-ER accumulation at a fission site in dsRed-Skl-labeled peroxisomes. Scale bar: $1 \mu \mathrm{m}$

Supplementary Movie 16|ER-associated actin accumulates at lysosomal fission sites. AC-ER accumulation at a fission site in LAMP1-mCherry-labeled lysosomes. Scale bar: $1 \mu \mathrm{m}$

Supplementary Movie 17|ER-associated actin accumulates at Golgi fission sites. AC-ER accumulation at a fission site in SiT-mApple-labeled Golgi. Scale bar: $1 \mu \mathrm{m}$

Supplementary Movie 18|ER-associated actin forms a network on the nucleus. 3D rendering of a U2OS cell expressing AC-ER reveals accumulation around the surface of the nucleus. Cyan: AC-ER. Orange: MitoTracker. 


\section{References}

1 Yang, C. \& Svitkina, T. M. Ultrastructure and dynamics of the actin-myosin II cytoskeleton during mitochondrial fission. Nat Cell Biol 21, 603-613, doi:10.1038/s41556-019-0313-6 (2019).

2 Jasnin, M. et al. Three-dimensional architecture of actin filaments in Listeria monocytogenes comet tails. Proc Natl Acad Sci U S A 110, 20521-20526, doi:10.1073/pnas.1320155110 (2013).

3 Rocchetti, A., Hawes, C. \& Kriechbaumer, V. Fluorescent labelling of the actin cytoskeleton in plants using a cameloid antibody. Plant Methods 10, 12, doi:10.1186/1746-4811-10-12 (2014).

4 Melak, M., Plessner, M. \& Grosse, R. Actin visualization at a glance. J Cell Sci 130, 525-530, doi:10.1242/jcs.189068 (2017).

5 Rapaport, D. Finding the right organelle. Targeting signals in mitochondrial outer-membrane proteins. EMBO reports 4, 948-952, doi:10.1038/sj.embor.embor937 (2003).

$6 \quad$ Friedman, J. R. et al. ER tubules mark sites of mitochondrial division. Science 334, 358-362, doi:10.1126/science.1207385 (2011).

7 Riedl, J. et al. Lifeact: a versatile marker to visualize F-actin. Nat Methods 5, 605-607, doi:10.1038/nmeth.1220 (2008).

8 Moore, A. S., Wong, Y. C., Simpson, C. L. \& Holzbaur, E. L. Dynamic actin cycling through mitochondrial subpopulations locally regulates the fission-fusion balance within mitochondrial networks. Nature communications 7, 12886, doi:10.1038/ncomms12886 (2016).

9 Manor, U. et al. A mitochondria-anchored isoform of the actin-nucleating spire protein regulates mitochondrial division. elife 4, doi:10.7554/elife.08828 (2015).

10 Ji, W. K., Hatch, A. L., Merrill, R. A., Strack, S. \& Higgs, H. N. Actin filaments target the oligomeric maturation of the dynamin GTPase Drp1 to mitochondrial fission sites. elife 4, e11553, doi:10.7554/eLife.11553 (2015).

11 Chakrabarti, R. et al. INF2-mediated actin polymerization at the ER stimulates mitochondrial calcium uptake, inner membrane constriction, and division. J Cell Biol 217, 251-268, doi:10.1083/jcb.201709111 (2018).

12 Korobova, F., Ramabhadran, V. \& Higgs, H. N. An actin-dependent step in mitochondrial fission mediated by the ER-associated formin INF2. Science 339, 464-467, doi:10.1126/science.1228360 (2013).

13 Korobova, F., Gauvin, T. J. \& Higgs, H. N. A role for myosin II in mammalian mitochondrial fission. Curr Biol 24, 409-414, doi:10.1016/j.cub.2013.12.032 (2014).

$14 \mathrm{Li}, \mathrm{S}$. et al. Transient assembly of F-actin on the outer mitochondrial membrane contributes to mitochondrial fission. J Cell Biol 208, 109-123, doi:10.1083/jcb.201404050 (2015).

15 De Vos, K. J., Allan, V. J., Grierson, A. J. \& Sheetz, M. P. Mitochondrial function and actin regulate dynamin-related protein 1-dependent mitochondrial fission. Curr Biol 15, 678-683, doi:10.1016/j.cub.2005.02.064 (2005).

16 Hatch, A. L., Ji, W. K., Merrill, R. A., Strack, S. \& Higgs, H. N. Actin filaments as dynamic reservoirs for Drp1 recruitment. Mol Biol Cell 27, 3109-3121, doi:10.1091/mbc.E16-03-0193 (2016).

17 Smirnova, E., Griparic, L., Shurland, D. L. \& van der Bliek, A. M. Dynamin-related protein Drp1 is required for mitochondrial division in mammalian cells. Mol Biol Cell 12, 2245-2256, doi:10.1091/mbc.12.8.2245 (2001).

18 Qin, J. Y. et al. Systematic comparison of constitutive promoters and the doxycycline-inducible promoter. PLoS One 5, e10611, doi:10.1371/journal.pone.0010611 (2010).

19 Rowland, A. A., Chitwood, P. J., Phillips, M. J. \& Voeltz, G. K. ER contact sites define the position and timing of endosome fission. Cell 159, 1027-1041, doi:10.1016/j.cell.2014.10.023 (2014).

20 Hoyer, M. J. et al. A Novel Class of ER Membrane Proteins Regulates ER-Associated Endosome Fission. Cell 175, 254-265 e214, doi:10.1016/j.cell.2018.08.030 (2018). 
21 Valm, A. M. et al. Applying systems-level spectral imaging and analysis to reveal the organelle interactome. Nature 546, 162-167, doi:10.1038/nature22369 (2017).

22 Gay, O. et al. RefilinB (FAM101B) targets filamin A to organize perinuclear actin networks and regulates nuclear shape. Proc Natl Acad Sci U S A 108, 11464-11469, doi:10.1073/pnas.1104211108 (2011).

23 Woroniuk, A. et al. STEF/TIAM2-mediated Rac1 activity at the nuclear envelope regulates the perinuclear actin cap. Nature communications 9, 2124, doi:10.1038/s41467-018-04404-4 (2018).

24 Kim, D. H., Cho, S. \& Wirtz, D. Tight coupling between nucleus and cell migration through the perinuclear actin cap. J Cell Sci 127, 2528-2541, doi:10.1242/jcs.144345 (2014).

25 Khatau, S. B., Kim, D. H., Hale, C. M., Bloom, R. J. \& Wirtz, D. The perinuclear actin cap in health and disease. Nucleus 1, 337-342, doi:10.4161/nucl.1.4.12331 (2010).

26 Khatau, S. B. et al. A perinuclear actin cap regulates nuclear shape. Proc Natl Acad Sci U S A 106, 1901719022, doi:10.1073/pnas.0908686106 (2009).

27 Chambliss, A. B. et al. The LINC-anchored actin cap connects the extracellular milieu to the nucleus for ultrafast mechanotransduction. Sci Rep 3, 1087, doi:10.1038/srep01087 (2013).

28 Schindelin, J. et al. Fiji: an open-source platform for biological-image analysis. Nat Methods 9, 676-682, doi:10.1038/nmeth.2019 (2012).

29 Borgese, N., Gazzoni, I., Barberi, M., Colombo, S. \& Pedrazzini, E. Targeting of a tail-anchored protein to endoplasmic reticulum and mitochondrial outer membrane by independent but competing pathways.

Molecular Biology of the Cell 12, 2482-2496 (2001).

30 Rohn, J. L. et al. Myo19 ensures symmetric partitioning of mitochondria and coupling of mitochondrial segregation to cell division. Curr Biol 24, 2598-2605, doi:10.1016/j.cub.2014.09.045 (2014).

31 Borgese, N., Gazzoni, I., Barberi, M., Colombo, S. \& Pedrazzini, E. Targeting of a tail-anchored protein to endoplasmic reticulum and mitochondrial outer membrane by independent but competing pathways. Mol Biol Cell 12, 2482-2496, doi:10.1091/mbc.12.8.2482 (2001).

32 Geiser, M., Cebe, R., Drewello, D. \& Schmitz, R. Integration of PCR fragments at any specific site within cloning vectors without the use of restriction enzymes and DNA ligase. BioTechniques 31, 88-90, 92 (2001). 